\title{
Bienestar psicológico en una muestra de estudiantes universitarios mexicanos
}

\section{Psychological well-being in a simple of mexican college students}

Jesús Ernesto Valenzuela Medina

Universidad de Sonora

\section{Resumen}

En el presente estudio se reporta la evaluación de la estructura factorial de una versión en español (Díaz, Rodríguez, Blanco, Moreno, Gallardo, Valle, \& van Dierendonck, 2006) de la Escala de Bienestar Psicológico, formulada por Carol Ryff (1989; Ryff \& Keyes, 1995) a partir de las respuestas de 1,060 estudiantes universitarios. El Análisis Factorial Confirmatorio (AFC) permite concluir que el ajuste del modelo a los datos es inapropiado, ya que no valida la estructura de seis dimensiones planteada originalmente, planteándose la hipótesis de que el cuestionario no es un modelo de medición apropiado para el constructo. La modalidad exploratoria del análisis factorial mediante ecuaciones estructurales permite identificar que el bienestar psicológico puede, para la muestra estudiada, ser explicado de forma satisfactoria mediante dos dimensiones: Crecimiento Personal, y Autoaceptación. Los indicadores de ajuste de dicho modelo, así como los de consistencia interna permiten concluir que la versión de 15 reactivos y dos dimensiones resulta una herramienta útil para el estudio de la adaptación experimentada por los estudiantes a la vida universitaria. Se requieren nuevas aplicaciones para calibrar el cuestionario.

Palabras clave: bienestar psicológico, modelo eudemónico, adaptación a la universidad, estudiantes universitarios, análisis factorial confirmatorio.

Nota del autor

Jesús Ernesto Valenzuela Medina, Departamento de Psicología y Ciencias de la Comunicación, Universidad de Sonora (UNISON).

La correspondencia en relación con este artículo debe dirigirse a Jesús Ernesto Valenzuela Medina, Departamento de Psicología y Ciencias de la Comunicación, UNISON, bulevar Luis Encinas y Rosales, s/n, colonia Centro, C. P. 83000, Hermosillo, Sonora, México.

Dirección electrónica: jevalenzuela@hotmail.com 


\begin{abstract}
This paper describes the assessment of the factor structure of the Spanish version (Díaz, Rodríguez, Blanco, Moreno, Gallardo, Valle, \& van Dierendonck, 2006) of the Ryff's (1989, Ryff \& Keyes, 1995) Psychological Well-being Scales, developed on the responses of 1060 college students. Confirmatory Factor Analysis (CFA) procedures applied allow to conclude that the model doesn't fit data properly, and it doesn't validate the 6 dimension structure originally formulated for the construct. In an exploratory mode, AFC procedures allowed to identify for the sample, two dimensions that explained Psychological Well-being: Personal Growth, and Self-Acceptance. Adjustment and internal consistency indices of the model showed that the 15 statements measure-grouped into the two dimensions or scales- is a good tool for studying students' adaptation to their life at college. Further studies are required to calibrate the questionnaire.
\end{abstract}

Keywords: psychological well-being, eudemonic model, adaptation to college life, college students, confirmatory factor analysis.

La sociedad actual, calificada por sociólogos contemporáneos como "de riesgo" (Beck \& Beck-Grensheim, 2003; Côté, 2005; Côté \& Levine, 2002; Giddens, 2002), se caracteriza por su condición de precariedad para la autodefinición; los individuos deben realizar elecciones en vez de sólo adoptar las tradiciones como vía para negociar y afirmar sus identidades. El contexto social en el que esta nueva transición a la vida adulta se lleva a cabo se caracteriza por las políticas orientadas al mercado y los estilos de vida basados en el consumo, que han venido a sustituir a las políticas orientadas a la comunidad y a los estilos de vida basados en la producción, propios de las sociedades industriales. Así, lo que hasta hace unos años eran problemas y asuntos que fueron tratados mediante gestión colectiva son ahora dejados a los individuos para que los resuelvan por sí mismos (Côté, 2002).
Las elecciones que deben realizar los individuos en esta sociedad, a veces llamada Alta Modernidad, Posmodernidad o Modernidad Tardía, no se hacen en condiciones estables y para mucha gente la complejidad de tales condiciones representa una fuente de ansiedad. La literatura sobre el desarrollo de la identidad personal en escenarios de transición, principalmente en jóvenes universitarios, tiene ya una tradición de más de 30 años en países anglosajones (Berzonsky, 1989; Côté, 2006; Côté \& Levine, 2002; Marcia, 1966, 1989; Schwartz, 2001).

Se han desarrollado propuestas teóricometodológicas diversas y se han identificado al menos dos tipos de estrategias con las que las personas abordan las tareas de formación de su identidad ante la exigencia de individualización propia de la sociedad contemporánea: una de aceptación pasiva, y otra de adaptación activa. A nivel individual, la respuesta pasiva parece 
más extendida e involucra el seguimiento irreflexivo de las contingencias prescritas institucionalmente, además del consentimiento de la manipulación de la identidad que caracteriza a la sociedad consumista-corporativista y a la cultura de las modas juveniles producidas por ésta. Dicha forma forma ha sido llamada individualización pasiva o, por defecto, y en términos comunes, se percibe como una estrategia de acción de mínimo esfuerzo. En contraste, otros individuos asumen una respuesta activa y se involucran en su propio crecimiento personal, lo que implica arriesgarse a tomar rutas de desarrollo y patrones de logro social y ocupacional más desafiantes. Este patrón activo es llamado individualización por desarrollo (Côté, 1996, 1997 ).

La mayoría de los jóvenes que viven la transición hacia la edad adulta en el contexto de sus estudios universitarios lo hace al interior de la burbuja de demora social, identificada por Erik Erikson. Ésta les dispensa, al menos momentáneamente, de la formulación de compromisos duraderos, alterando su percepción de bienestar. Desde una perspectiva psicosocial, inaugurada por Erikson en su interés por explicar la identidad personal al inicio de la vida adulta, el estudio de los procesos de formación y afirmación de ésta resulta crucial.

Con un enfoque centrado en los procesos de exploración y compromiso, Michel Berzonsky (1989, 1997, 2008) propone que los estilos de identidad representan la clave para entender la formación y mantenimiento de la identidad en esta fase de transición. Siguiendo esta tradición de investigación, Berzonsky y otros (Adams, Berzonsky, \& Keating, 2006; Berzonsky, 2003; Berzonsky \& Kuk, 2000; Nurmi, Berzonsky, Tammi, \& Kinney, 1997), han estudiado las relaciones entre los estilos de identidad, variables de la persona y el constructo de bienestar.

En este documento se presenta un trabajo en el que se estudió la estructura factorial del constructo de Bienestar Psicológico. Se reportan los resultados de este estudio, perteneciente a un trabajo más amplio en el cual se exploran las relaciones entre los estilos de identidad y el bienestar psicológico en estudiantes universitarios mexicanos.

\section{El bienestar psicológico}

El concepto de bienestar ha sido motivo de estudio desde distintas perspectivas. En la literatura se identifican dos grandes tradiciones: la hedónica, relacionada con la felicidad, y la eudemónica, relacionada con el desarrollo y expresión del potencial humano. Keyes, Shmotkin y Ryff (2002) señalan que el bienestar subjetivo representa el interés de la primera, en tanto que el bienestar psicológico el de la segunda. Este enfoque ha sido identificado como representativo de la llamada psicología positiva. El núcleo de la visión positiva del funcionamiento psicológico saludable considera como hipótesis que la experiencia de bienestar contribuye al funcionamiento efectivo, no sólo de múltiples sistemas orgánicos cuando hablamos de salud -lo que ayuda a no sucumbir ante la enfermedad y también a recuperarse de 
ella-, sino que el beneficio de tal experiencia se extiende a los diversos aspectos relacionales de las personas.

Al considerar la creación de su modelo de funcionamiento positivo como alternativa a las visiones centradas en el déficit o en aspectos relativos a patologías psicológicas, Ryff (1989) plantea que el bienestar psicológico implica "funcionar o estar bien" a diferencia de solamente "sentirse bien". Para sustentar el modelo, retoma ideas de pensadores representativos de tres áreas o campos especializados: el enfoque de ciclo vital que explica la continuidad del desarrollo humano durante la vida; el enfoque humanista aplicado a la práctica clínica y la psicoterapia; y el enfoque positivo para la definición de la salud mental. En la tabla 1 se resumen las ideas principales en las cuales se basa su formulación así como los autores que considera para su justificación.

Tabla 1

Bases conceptuales del enfoque positivo del Bienestar Psicológico formulado por Carol Ryff

\begin{tabular}{lll}
\hline \multicolumn{1}{c}{ Tradición } & \multicolumn{1}{c}{ Autores } & Ideas \\
\hline Ciclo vital & $\begin{array}{l}\text { Erik Erikson, Karl Bühler, Ber- } \\
\text { nice Neugarten }\end{array}$ & $\begin{array}{l}\text { El bienestar es producto y a la } \\
\text { vez factor de una progresión y } \\
\text { un continuo crecimiento. }\end{array}$ \\
$\begin{array}{lll}\text { Psicoterapia humanista y personalidad } \\
\text { Enfoque positivo de la salud mental }\end{array}$ & $\begin{array}{l}\text { Carl Rogers, Abraham Maslow, } \\
\text { Gordon Allport }\end{array}$ & $\begin{array}{l}\text { El sentido de control y autonomía } \\
\text { alcanzada, así como la impor- } \\
\text { tancia del proyecto vital hacia el } \\
\text { crecimiento. }\end{array}$ \\
& Marie Jahoda & $\begin{array}{l}\text { Tendencia hacia el crecimiento } \\
\text { y la actualización constante de }\end{array}$ \\
& & $\begin{array}{l}\text { uno mismo a través del control } \\
\text { del entorno. }\end{array}$
\end{tabular}

Nota: elaboración propia a partir de Carol Ryff (1989), y Ryff y Singer (2006).

Con todos estos antecedentes, para el modelo que recupera la tradición eudemónica clásica de Aristóteles, bienestar es un constructo donde se expresa el sentir positivo y el pensar constructivo del ser humano acerca de sí mismo, definido por su naturaleza subjetiva, vivencial y relacional. Vincula aspectos particulares del funcionamiento físico, psíquico y social; además se considera que posee elementos reactivos o transitorios, es decir, expresiones de la esfera emocional, así como expresiones de lo cognitivo y de lo valorativo como elementos estables, ambos en estrecha relación y muy influidos por la personalidad como sistema de interacciones complejas. Asimismo, por las circunstancias medioambientales, especialmente las más estables, por ejemplo, las diferencias socio-demográficas, el momento del ciclo vital, las condiciones materiales de vida, nivel educacional, ocupación, grupo social, 
principalmente (García-Viniegras \& González, 2000). Este constructo es relacional también porque vincula las experiencias y el momento presente con una proyección hacia el futuro. Representa pues, un recurso psicosocial muy importante para hacer frente a las circunstancias, y resulta crucial particularmente ante aquellas relacionadas con transiciones significativas en la vida de las personas (Vera \& Valenzuela, 2012).

En dicho contexto, es de nuestro interés estudiar las transiciones que enfrentan los jóvenes que ingresan a las instituciones de educación superior a realizar sus estudios. Son diversas las demandas y presiones característicos de esta transición experimentada por los jóvenes que tienen la suerte de vivirla, entre éstos se hallan las familiares, las del grupo de pares, las expectativas personales, las que se relacionan con la propia vida institucional, y las del gremio profesional al que se aspira a integrarse, principalmente. De acuerdo a Roe (2005), la forma o estrategia con la que cada joven responde a estas demandas irá configurando su trayectoria por la institución e irá conformando y reconfigurando su identidad como individuo, como estudiante y como ciudadano. Sus percepciones de bienestar representan un peso específico elevado en esta ecuación. Desde una perspectiva psicosocial, lo que está en juego en dicha esta transición es la adaptación e integración de los jóvenes a una cierta vida institucionalizada, un proceso que consiste en descubrir y asimilar, en el contacto con las rutinas de las prácticas escolares, conocimientos tácitos, normas y valores mediante los que descifrarán la enseñanza superior y se apropiarán de ella (De Garay, 2004, 2005).

El estudio de esta transición que experimentan los jóvenes implica la identificación de los modos en que éstos se representan la tarea de estudiar (identidad como estudiantes, dimensión correspondiente a la identidad personal), las estrategias que realizan para lograrlo (roles asumidos), sus atribuciones de éxito y fracaso, y sus valoraciones de los resultados alcanzados. En esta interacción dinámica es donde se manifiesta la subjetividad que buscamos capturar y explorar, al tratar de entender sus particularidades. Contextualizada así, nos interesan las percepciones de bienestar, ya que seguramente sufrirán transformaciones asociadas con las experiencias vividas durante su estancia en la universidad (ver Adams et al., 2006; Chickering \& Reisser, 1993; De Garay, 2004, 2005; Pascarella \& Terenzini, 2005).

El estudio de la subjetividad implicada en las percepciones de bienestar desde el modelo formulado por Ryff (1989), parte de la idea según la cual el bienestar se relaciona con el funcionamiento en las siguientes seis dimensiones: Autoaceptación, caracterizada por la actitud positiva hacia uno mismo, abarcando tanto los aspectos positivos como negativos; Autonomía, relacionada con la independencia y la capacidad para manejar la presión social; Control del entorno, que abarca el manejo constructivo de las presiones simultáneas de la cotidianidad; Crecimiento personal, incluye la percepción de crecimiento y desarrollo continuos y la satisfacción con- 
comitante; Relaciones positivas con los demás, implica el cultivo de relaciones cordiales, de soporte y cuidado con los demás; y finalmente, Propósito en la vida, relacionada con el sentido de orientación a metas significativas.

En el reporte de Díaz et al. (2006) y en el de van Dierendonck, Díaz, Rodríguez, Blanco y Moreno (2008), se analizan distintas versiones de cuestionarios diseñados para operacionalizar el constructo y se discuten sus características y limitaciones. La versión reportada por Díaz et al. (2006) es la primera elaborada en nuestro idioma y fue validada con población española, por lo cual decidimos realizar un estudio con estudiantes universitarios mexicanos para explorar si el constructo de bienestar psicológico con el que fue elaborada resulta de utilidad para comprender la transición de la que hablamos en la sección anterior.

Así pues, el objetivo del estudio fue evaluar la estructura factorial que sirve de base al cuestionario de Díaz et al. (2006), construido con la lógica del modelo de bienestar psicológico de Carol Ryff (1989).

\section{Método}

\section{Participantes}

En un estudio más amplio (Valenzuela, 2011), orientado a explorar la formación de la identidad en estudiantes universitarios, participaron 1,100 estudiantes de distintos semestres, excepto de primer año, inscritos en diversas carreras, en tres instituciones de educación superior del estado de Sonora, México. Para el presente reporte se consideraron sólo 1,060 cuestionarios por contener información completa, útil para el análisis. El 64\% de la muestra lo representan las mujeres, en tanto la edad promedio en la muestra fue de 22 años. Más de la mitad reportó estar inscrita, con estatus académico regular, entre el tercero y el séptimo semestre de su carrera, el resto completaba cursos correspondientes a distintos semestres y su estatus era de estudiantes irregulares.

\section{Variables e instrumentos}

El constructo de interés en este estudio fue el bienestar psicológico de los estudiantes. El modelo de medición empleado se basó en las respuestas a las Escala de Bienestar Psicológico (Díaz et al., 2006), versión en español de 29 enunciados, creada a partir de la versión original en inglés (Ryff, 1989). Para nuestro estudio, analizamos la definición conceptual de las escalas y la redacción de los enunciados de la versión original en inglés, y después de su traducción-adaptación así como de un pilotaje para ajustar la redacción final, realizada con un grupo de estudiantes avanzados de psicología, decidimos añadir uno a la dimensión crecimiento personal de la versión española, para conformar el cuestionario definitivo con un total de 30 enunciados, cinco por dimensión. Se mantuvo la escala de respuestas tipo Likert con seis anclajes, que va de total desacuerdo (1) a total acuerdo (6), en la cual puntajes mayores describen niveles superiores de bienestar. En la versión de Díaz et al. (2006) y en nuestro cuestionario se refleja 
la estructura de seis factores propuesta en el original en inglés. En este documento sólo se reporta el análisis que evaluó la validez de dicha estructura factorial mediante los procedimientos del AFC propuestos por Byrne (2010).

\section{Materiales}

Al final de este escrito, en un apéndice, se presenta el cuestionario de 30 reactivos empleado para la medición de las percepciones de bienestar de los jóvenes universitarios. Para el estudio, en sus respectivas aulas y en horarios de disponibilidad durante sus clases, se entregó a cada participante un sobre individual que contenía el cuestionario. En dicho levantamiento se aplicaron también otros cuestionarios; uno para medir el constructo de estilo de identidad, y otro para obtener información de identificación personal y otras estimaciones sobre el grado de adaptación a la vida universitaria. En este último, los estudiantes leían información descriptiva sobre el estudio y en caso de acordar firmaban su consentimiento para participar.

\section{Procedimiento}

Tras obtener la autorización institucional para contactar a los estudiantes se procedió de la siguiente manera:

Levantamiento de la información. Al asistir a las aulas en los horarios autorizados, se presentó información oral a los estudiantes sobre el estudio, se les indicó el propósito del mismo, a saber, obtener información útil para la planificación del programa de tutoría de su respectiva institución. Después de escuchar la información y la lectura del texto descriptivo del estudio se les pidió firmar su consentimiento de participación informado; una vez aceptado, se les pidió a quienes así lo decidieron resolvieran los cuestionarios, primero la cédula de información, luego el cuestionario de bienestar psicológico de este estudio, y al final el de estilo de identidad. El aplicador permanecía en el aula para resolver dudas específicas y al término recogía y organizaba los paquetes para su posterior revisión.

Organización de la información. Un ayudante fue capacitado y se encargó de la captura electrónica de los cuestionarios y de la preparación de la base de datos electrónica para su procesamiento. En la versión usada en este estudio se mantuvo casi sin modificación la redacción de los reactivos del cuestionario español (ver apéndice al final).

Análisis de la información. Después de diversos análisis preliminares de la base de datos, se procedió a realizar la evaluación de la estructura factorial del cuestionario. Para ello se siguieron los procedimientos de AFC formulados por Tabachnick y Fidell (2007), y principalmente por Byrne (2010). Se evaluó primero el correspondiente a la estructura original propuesta, conformada por seis factores, y luego fueron puestas a prueba distintas estructuras. Para tal fin, se usó el módulo de AMOS del SPSS Versión 21 (Arbuckle, 2012). Primero se utilizó una estrategia confirmatoria para evaluar la validez de la estructura de seis dimensiones, más tarde se empleó una estrategia exploratoria, donde se probaron diversos modelos hasta 
identificar una solución con indicadores de ajuste apropiados. Con base en el análisis de la normalidad univariada y multivariada de la distribución, se siguió la recomendación de Browne, de emplear el método de estimación de distribución libre de curva en vez del de máxima verosimilitud (Byrne, 2010; Hair, Anderson, Tatham, \& Black, 1999). También se tomó en cuenta el criterio propuesto por West, Finch y Curran (1995, citado en Byrne, 2010), ya que la muestra superó el mínimo de 1,000 casos.

\section{Resultados}

Aunque se evaluaron varios modelos, en la tabla 2 se presentan los indicadores de ajuste de sólo dos de ellos: los de la solución de seis factores, cuyos índices resultaron claramente inapropiados, lejos de los estándares de ajuste recomendados; y los de la mejor solución identificada, tanto empírica como conceptualmente, que corresponde a una estructura de dos factores, conformada por la mitad de los reactivos del cuestionario empleado. Ambos factores mostraron una correlación positiva baja entre sí $(r=.102)$, lo que permite pensar en la viabilidad de estas dos dimensiones independientes que conforman el constructo de bienestar en esta muestra de jóvenes universitarios.

Tabla 2

Estadísticas de ajuste para modelos alternativos puestos a prueba en el estudio con estudiantes universitarios mexicanos $(N=1060)$

\begin{tabular}{|c|c|c|c|}
\hline Modelo & Escala y reactivos & $\begin{array}{c}\text { Alfa de } \\
\text { Cronbach }\end{array}$ & Estadísticos de ajuste del modelo \\
\hline \multirow{6}{*}{6 factores } & Autoaceptación $(1,7,12,17,24)$ & .473 & \multirow{6}{*}{$\begin{array}{l}X^{2}=4713.470 ; g I=390 ; \\
C F I=.632 ; \mathrm{PCFI}=.567 ; \text { SRMR = .1506; } \\
\text { RMSEA =.102; IC (.100-.105); PCLOSE } \\
=.000\end{array}$} \\
\hline & Relaciones c/otros $(2,8,13,22,25)$ & .491 & \\
\hline & Autonomía $(3,4,9,18,23)$ & .477 & \\
\hline & Dominio d/entorno $(5,10,14,19,29)$ & .496 & \\
\hline & Propósito E/vida $(6,11,15,16,20)$ & .584 & \\
\hline & Crec. personal $(21,26,27,28,30)$ & .407 & \\
\hline \multirow{4}{*}{2 factores } & Crecimiento personal & & \multirow{4}{*}{$\begin{array}{l}X^{2}=265.988 ; g l=89 ; \\
C F I=.859 ; \mathrm{PCFI}=.728 ; \text { SRMR = .051; } \\
\text { RMSEA = .043; IC (.037-.049); PCLOSE } \\
=.966\end{array}$} \\
\hline & $(17,14,20,11,18,13,21)$ & .809 & \\
\hline & Autoaceptación & & \\
\hline & $(15,16,7,5,19,3,24,6)$ & .862 & \\
\hline
\end{tabular}

Nota: Elaboración propia a partir de la salida de AMOS 21 de SPSS. CFI = índice de ajuste comparativo; SRMR = raíz cuadrática media de residuales; RMSEA = raíz cuadrática media del error de aproximación; IC = Intervalo de confianza del RMSEA; PCLOSE = probabilidad de adecuación del ajuste del modelo a los datos. 
En la solución de dos factores podemos observar que el Índice de Ajuste Comparativo (CFI) se halla por debajo del rango de aceptación propuesto por $\mathrm{Hu}$ y Bentler (1999, citado en Byrne, 2010) de .95, por lo cual el ajuste del modelo a los datos es pobre; sin embargo, al evaluar los otros índices podemos ver que la solución presenta potencial explicativo. La parsimonia del modelo, considerada en el valor del PCFI (.728), resulta adecuada para el tamaño de la muestra y la cantidad de parámetros estimados. El valor de la raíz cuadrática media de residuales $(S R M R)$, relativamente bajo
(.051); el valor de la raíz cuadrática media del error de aproximación (RMSEA), por debajo del .05 recomendado por Browne y Cudeck, (1993, citado en Byrne, 2010, p. 80), así como el nivel de precisión de éste, expresado en la relativamente corta amplitud de su intervalo de confianza (IC); además del valor de PCLOSE muy superior al límite de .50 recomendado por Joreskög y Sörbom (citado en Byrne, 2010, p. 81), permiten considerar con optimismo, aunque no sin reservas, el ajuste de este modelo a los datos de nuestra muestra. En la figura 1 se muestra la estructura bifactorial descrita.
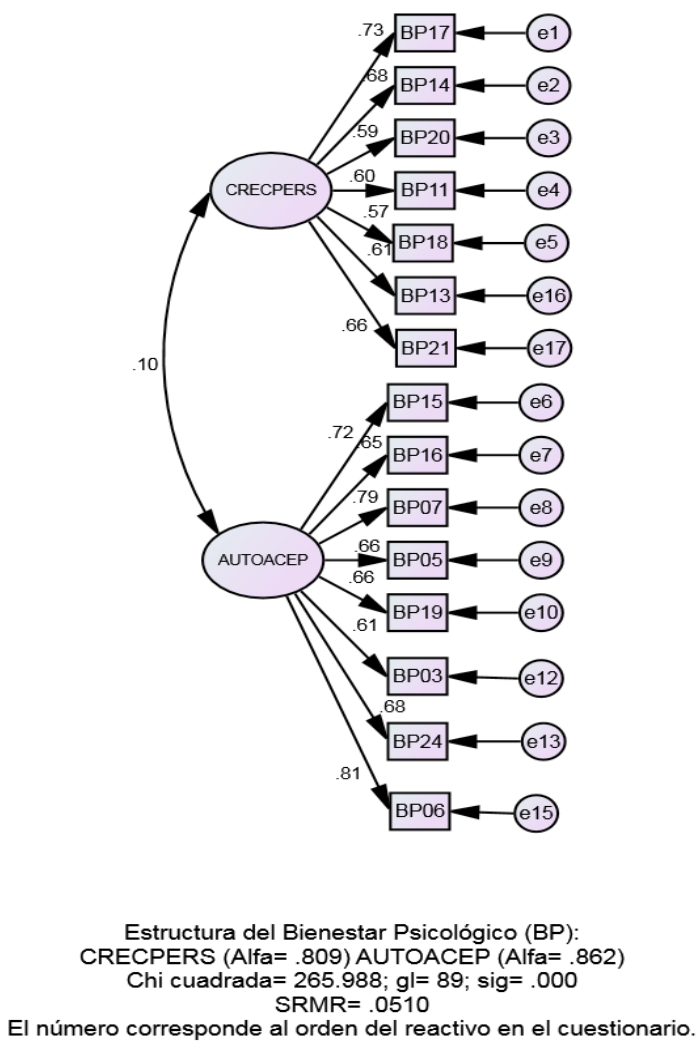

Figura 1. Modelo bifactorial de bienestar psicológico en una muestra de estudiantes universitarios mexicanos ( $N=1060$, valores estandarizados). 


\section{Tabla 3}

Cargas factoriales y estadísticas descriptivas para las dimensiones resultantes del análisis exploratorio en el estudio con universitarios mexicanos

\begin{tabular}{|c|c|c|c|c|c|c|}
\hline \multirow{2}{*}{ Dimensión } & \multirow{2}{*}{ Reactivo } & \multirow{2}{*}{$\begin{array}{c}\text { Cargas } \\
\text { factoriales }\end{array}$} & \multicolumn{4}{|c|}{ Estadísticas descriptivas de las dimensiones } \\
\hline & & & $M$ & $D E$ & Min. & Máx. \\
\hline Crecimiento & 17 & .729 & \multirow{7}{*}{4.987} & \multirow{7}{*}{0.167} & \multirow{7}{*}{4.809} & \multirow{7}{*}{5.270} \\
\hline \multirow{6}{*}{ personal } & 14 & .677 & & & & \\
\hline & 20 & .590 & & & & \\
\hline & 11 & .601 & & & & \\
\hline & 18 & .566 & & & & \\
\hline & 13 & $.615)$ & & & & \\
\hline & 21 & .662 & & & & \\
\hline \multirow[t]{8}{*}{ Autoaceptación } & 15 & .723 & \multirow{8}{*}{4.016} & \multirow{8}{*}{0.240} & \multirow{8}{*}{3.692} & \multirow{8}{*}{4.347} \\
\hline & 16 & .652 & & & & \\
\hline & 7 & .795 & & & & \\
\hline & 5 & .665 & & & & \\
\hline & 19 & .656 & & & & \\
\hline & 3 & .610 & & & & \\
\hline & 24 & .683 & & & & \\
\hline & 6 & .806 & & & & \\
\hline
\end{tabular}

Correlación entre las dimensiones: $r=.102$

Nota: elaboración propia a partir de la salida de SPSS 21. Ver enunciado correspondiente a cada número de reactivo en el apéndice.

En la tabla 3 se muestra la conformación de las dimensiones resultantes, junto con otros datos.

\section{Discusión}

El constructo multidimensional de bienestar psicológico, desde la perspectiva positiva del enfoque eudemónico, resulta una aproximación viable para apoyar la explicación de los procesos de formación, mantenimiento o cambio de la identidad personal, en donde la percepción subjetiva de bienestar resulta un componente muy importante (Adams et al., 2006; Berzonsky, 2003; Berzonsky \& Kuk, 2000; Kerpelman, Pittman, \& Adler-Baeder, 2008). Sin embargo, el modelo de medición original de seis factores empleado en este estudio no pasó la prueba empírica. Diversos estudios han reportado dificultades para validar la compleja estructura de seis factores que Ryff (1989) propuso para explicar su visión positiva del bienestar 
psicológico (ver van Dierendonck et al., 2008 para una revisión de las críticas). Es posible que parte de la dificultad provenga de la escasa validez factorial y la falta de consistencia interna de la versión original de las escalas, mismas que fueron derivadas teóricamente por Ryff (1989) a partir de su revisión de la literatura con enfoque de psicología y psicoterapia positivas. Un señalamiento concreto encontrado en la literatura se refiere al traslape -en su modelo original-, de cuatro de las seis dimensiones en una sola: Crecimiento personal; Propósito en la vida; Autoaceptación; y Dominio del entorno. Otro señalamiento tiene relación con la naturaleza ordinal de las escalas empleadas y el uso de procedimientos analíticos que suponen medidas continuas, lo cual produce sesgos en la estimación.

La propuesta de Díaz et al. (2006) representa un esfuerzo por mejorar las características psicométricas del modelo de medición del constructo, por lo tanto, decidimos usarla para evaluar su potencial con una muestra de jóvenes universitarios mexicanos. Nuestros resultados difieren de los reportados por los autores españoles. En su artículo, Díaz et al. (2006) reportan valores de Alfa de Cronbach entre $.70 \mathrm{y}$ .84 para su versión de 29 reactivos y seis escalas; y una chi cuadrada de $615.76 ; 345$ grados de libertad; un CFI de 0.95; un NNFI de 0.94; un $S M R M$ de 0.05 ; y un RMSEA de 0.04 para un modelo de seis factores primarios y uno solo de segundo orden, con un método de estimación de Máxima Verosimilitud. Los indicadores de ajuste y los de consistencia interna de las respuestas al cuestionario de 30 preguntas, al agruparse en el modelo de seis dimensiones que nosotros empleamos, resultaron muy insatisfactorios, lo que nos llevó a explorar distintas estructuras para identificar aquella que arrojara una solución apropiada para estudiar las percepciones de bienestar de nuestros jóvenes.

Así, la expresión de bienestar entre nuestros 1,060 universitarios presentó una estructura conformada por las dimensiones que decidimos nombrar Crecimiento personal y Autoaceptación, usando la terminología original. Dado el carácter exploratorio del procedimiento empleado para identificarlas, decidimos llamarlas así al interpretar el contenido expresado en los enunciados que las conformaron, aunque éstos pertenecieran a distintas dimensiones en el estudio original. No obstante que nuestro juicio se basa en el conocimiento de la definición conceptual de las escalas, dado por Ryff (1989), entendemos que una limitación del estudio vendría de nuestra interpretación del contenido de los enunciados, aspecto relacionado con la problemática del modelo de medición. Este último ha sido derivado del concepto de bienestar de la autora, que como ya comentamos, ha sido criticado metodológicamente en diversas ocasiones (van Dierendonck et al., 2008). En la siguiente tabla se incluyen estas definiciones para permitiré al lector establecer un juicio sobre la pertinencia de nuestra interpretación. 
Tabla 4

Definición conceptual de las dimensiones de bienestar psicológico en el estudio con universitarios mexicanos

\begin{tabular}{|c|c|}
\hline Dimensión & Definición original de Ryff (1989) \\
\hline \multirow[t]{2}{*}{ Autoaceptación } & $\begin{array}{l}\text { Puntajes altos: describen a quienes poseen una actitud positiva hacia sí mismos, } \\
\text { que reconocen y aceptan los múltiples aspectos positivos o negativos que los } \\
\text { caracterizan, asimismo, acepta positivamente su pasado. }\end{array}$ \\
\hline & $\begin{array}{l}\text { Puntajes bajos: describe a quienes se sienten insatisfechos consigo mismos y de- } \\
\text { cepcionados con su pasado; quienes no aceptan algunas de sus características y } \\
\text { quisieran ser alguien distinto a quienes son. }\end{array}$ \\
\hline \multirow[t]{2}{*}{ Crecimiento personal } & $\begin{array}{l}\text { Puntajes altos: describen a quienes consideran y aceptan un sentido de continui- } \\
\text { dad y expansión en su propio desarrollo; quienes se muestran abiertos a nuevas } \\
\text { experiencias al mostrar conciencia de su potencial a lo largo del tiempo, les brinda } \\
\text { un sentido de autoconocimiento y efectividad. }\end{array}$ \\
\hline & $\begin{array}{l}\text { Puntajes bajos: describen a quienes muestran un sentido de estancamiento, care- } \\
\text { cen de la visión de continuidad y expansión; quienes se muestran pesimistas y } \\
\text { aburridos con la vida y se sienten incapaces de desarrollar nuevas actitudes y } \\
\text { conductas. }\end{array}$ \\
\hline
\end{tabular}

Nota: Traducción del original de Ryff (1989), elaboración personal.

Desde nuestra perspectiva, no carece de lógica considerar que la actitud positiva hacia uno mismo (Autoaceptación), que en promedio reportan los estudiantes $($ Media $=4.01)$, se relacione con un alto sentido de realización o Crecimiento personal $($ Media $=4.99)$. La baja correlación resultante entre ambas dimensiones $(r=.102)$ en el modelo, nos indica la relativa independencia de ambas en la experiencia vivencial de los jóvenes en su adaptación a la vida universitaria. Resultados preliminares en la prueba de un modelo estructural, donde se incluyeron las relaciones entre estas dos dimensiones con las variables Ajuste a la universidad, Satisfacción con la carrera elegida, y Valoración de la capacidad para estudiarla, han mostrado efectos directos elevados de la dimensión Cre- cimiento personal sobre los puntajes resultantes de un índice de ajuste (AJINDEX), conformado por estimaciones de facilidad para la adaptación a la institución, a la carrera, a los profesores y a los compañeros. También efectos directos y altos sobre la percepción de capacidad para estudiar la carrera, además de otros efectos indirectos relevantes para entender el proceso de adaptación de los jóvenes. En dicho modelo, la dimensión Autoaceptación, aunque correlacionada con la dimensión de Crecimiento personal $(r=.142)$ no mostró efectos ni directos ni indirectos sobre las variables de ajuste. El modelo de medición descrito hasta aquí deberá seguir su proceso de prueba, si consideramos que este tipo de estudio corresponde a lo que García-Viniegras y González (2000) clasifican 
como enfoque dimensional del bienestar, dado que nuestras indagaciones con base en la escala original no apoya, la idea del enfoque de interpretación global del bienestar.

Nos anima la perspectiva de estudiar con un enfoque psicosocial la transición de los jóvenes universitarios (Vera \& Valenzuela, 2012), con el fin de aportar información que resulte relevante para fortalecer los programas de tutoría y orientación en las IES, pues como señalan diversos autores (De Garay, 2004, 2005; Silva, 2011; Valenzuela, 2011), es preciso que las instituciones sustenten los esfuerzos de atención a los estudiantes en el conocimiento de su experiencia de adaptación a la vida universitaria. El conocimiento de la dimensión subjetiva correspondiente, a sus percepciones de bienestar psicológico, resulta de especial relevancia. Este estudio muestra la preocupación por desarrollar instrumentos válidos y confiables para medir dicho constructo y emplearlo en la formulación y puesta a prueba de modelos explicativos de la transición a la que hemos hecho referencia.

\section{Referencias}

Adams, G., Berzonsky, M., \& Keating, L. (2006). Psychosocial resources in first-year university students: The role of identity processes and social relationships. Journal of Youth and Adolescence, 35(1), 81-91.

Arbuckle, J. L. (2012). IBM SPSS, Amos 21 User's Guide. Crawfordville, FL: Amos Development Corporation,
Beck, U., \& Beck-Grensheim, E. (2003). La individualización. El individualismo institucionalizado y sus consecuencias sociales $y$ políticas. Barcelona: Paidós Ibérica.

Côté, J. (1996). Sociological perspectives on identity formation: The cultural identity link and identity capital. Journal of Adolescence, 19, 419-430.

Côté, J. (1997). An empirical test of the identity capital model. Journal of Adolescence, 20, 577-597.

Côté, J. (2002). The role of identity capital in the transition to adulthood: The individualization thesis examined. Journal of Youth Studies, $5(2), 117-134$.

Côté, J. (2005). Identity capital, social capital, and the wider benefits of learning: Generating resources facilitative of social cohesion. London Review of Education, 3(3), 221-237.

Côté, J. (2006). Identity studies: How close are we to developing a Social Science of Identity? An Appraisal of the field. Identity: An International Journal of Theory and Research, 6(1), 3-25.

Côté, J., \& Levine, Ch. (2002). Identity formation, agency, and culture: A social psychological synthesis. Hillsdale, NJ: Lawrence Erlbaum.

Berzonsky, M. (1989). Identity style: Conceptualization and measurement. Journal of Adolescent Research, 4, 267-281.

Berzonsky, M. (1997). Identity development, control theory, and self-regulation: An in- 
dividual differences perspective. Journal of Adolescence Research, 12, 347-353.

Berzonsky, M. (2003). Identity style and wellbeing: Does commitment matter? Identity, 3(2), 131-142.

Berzonsky, M. (2008). Identity formation: The role of identity processing style and cognitive processes. Personality and Individual Differences, 44, 643-653.

Berzonsky, M., \& Kuk, L. (2000). Identity status, identity processing style, and the transition to university. Journal of Adolesacent Research, 15(1), 81-98.

Byrne, B. (2010). Structural Equation Modeling with AMOS. Basic concepts, applications, and programming ( $2^{\text {nd }}$ ed.). New York, NY: Routledge.

Chickering, A., \& Reisser, L. (1993). Education and Identity, (2nd ed.). San Francisco, CA: Jossey-Bass.

De Garay, A. (2004). Integración de los jóvenes en el sistema universitario. Barcelona/México: Pomarés.

De Garay, A. (2005). En el camino de la universidad. México: UAM-EON.

Díaz, D., Rodríguez, C. R., Blanco, A., Moreno, J. B., Gallardo, I., Valle, C., \& van Dierendonck, D. (2006). Adaptación española de las escalas de bienestar psicológico de Ryff. Psicothema, 18(3), 572-577. Recuperado de https://www.uam.es/gruposinv/esalud/ Articulos/Psicologia\%20Positiva/2006Wellbeing\%20psicothema.pdf
García-Viniegras, C. R. V., \& González, B. I. (2000). La categoría bienestar psicológico, su relación con otras categorías sociales. Rev Cub Med Gen Integr, 16(6), 586-592.

Giddens, A. (2002). Modernidade e Identidade. Rio de Janeiro: Jorge Zahar Editor.

Hair J., Anderson R., Tatham R., \& Black W. (1999). Análisis Multivariante (5a. ed.). Madrid: Pearson.

Kerpelman, J. L., Pittman, J., \& Adler-Baeder, F. (2008). Identity as a moderator of intervention-related change: Identity style and adolescents' responses to relationships education. Identity, 8(2), 151-171.

Keyes, C. L., Shmotkin, D., \& Ryff, C. D. (2002). Optimizing well-being: The empirical encounter of two traditions. Journal of Personality and Social Psychology, 82(6), 1007-1022.

Marcia, J. (1966). Development and validation of ego identity status. Journal of Personality and Social Psychology, 5, 551-558.

Marcia, J. (1989). Identity and intervention. Journal of Adolescence, 12, 401-410.

Nurmi, J., Berzonsky, M. Tammi, K., \& Kinney, A. (1997). Identity processing orientation, cognitive and behavioral strategies and wellbeing. International Journal of Behavioral Development, 21(3), 555-570.

Pascarella, E., \& Terenzini, P. (2005). How college affects students, Vol. 2, a third decade of research. San Francisco, CA: Jossey-Bass. Roe, M. (2005). Negotiating the freshmen year: Challenges and strategies among first-year 
college students. Journal of College Student Development, 46(3), 296-316.

Ryff, C. (1989). Happiness is everithing, or Is it? Explorations on the meaning of psychological well-being. Journal of Personality and Social Psychology. 57, 1069-1081.

Ryff, C., \& Keyes, C. (1995). The structure of psychological well-being revisited. Journal of Personality and Social Psychology, 69, 719-727.

Ryff, C., \& Singer, B. (2006). Best news yet on the six-factor modelo of well-being. Social Science Research, 35, 1103-1119.

Schwartz, J. (2001). The evolution of Eriksonian and neo-Eriksonian identity theory and research: A review and integration. Identity, 1(1), 7-58.

Silva, M. (2011). El primer año universitario. Un tramo crítico para el éxito académico. Perfiles Educativos, 33, 102-114. Recuperado de: http://www.redalyc.org/src/inicio/ArtPdfRed.jsp?iCve $=13221258010$

Tabachnick, B., \& Fidell, L. (2007). Using Multivariate Statistics (5th ed.). Boston, MA: Pearson.

Valenzuela, J. (2011). Formación de identidad en estudiantes universitarios: Un diseño combinado secuencial explicativo (Tesis doctoral inédita). Universidad Autónoma de Sinaloa, Culiacán de Rosales, Sinaloa, México.

Van Dierendonck, D., Díaz, D., Rodríguez, C. R., Blanco, A., \& Moreno, J. B. (2008). Ryff's Six-Factor model of psychological well-being: A spanish exploration. Soc Indic Res, 87, 473-479.

Vera, J., \& Valenzuela, J. (2012). El concepto de identidad como recurso para el estudio de transiciones. Psicología \& Sociedades, 24(2), 272-282.

Recibido el 17 de septiembre de 2014 Revisado el 13 de octubre de 2014 Aceptado el 17 de diciembre de 2014 


\section{Apéndice}

Cuestionario de Bienestar Psicológico empleado en el estudio con estudiantes universitarios. Se destacan en negritas los enunciados redactados en sentido negativo, y en cursivas los que conformaron las dimensiones resultantes. $\mathrm{AA}=$ Autoaceptación; $\mathrm{RD}=$ Relaciones con los demás; $\mathrm{AU}=$ Autonomía; $\mathrm{DE}=$ Dominio del Entorno; $\mathrm{PV}=$ Propósito en la vida; $\mathrm{CP}=$ Crecimiento personal. $\mathrm{La}$ escala de respuestas empleada va de en total desacuerdo (1) hasta total Acuerdo (6).

1 Cuando repaso la historia de mi vida estoy contento con cómo han resultado las cosas.

2 A menudo siento que tengo pocos amigos íntimos c/quienes compartir mis preocupaciones.

3 No tengo miedo de expresar mis opiniones aunque sean opuestas a las de la mayoría de la gente.

4 Me preocupa cómo evalúan los demás las elecciones que he hecho en mi vida.

5 Me resulta difícil dirigir mi vida hacia un camino que me satisfaga.

Disfruto al trabajar para hacer realidad los planes que he hecho.

DE

En general, me siento seguro y a gusto conmigo mismo.

PV

8 No tengo muchas personas que quieran escucharme cuando necesito hablar.

9 Tiendo a preocuparme sobre lo que otra gente piensa de mí.

RD

Soy una persona activa al realizar los proyectos que me propuse.

Si tuviera la oportunidad hay muchas cosas de mí mismo que cambiaría.

PV

13 Siento que mis amistades me aportan muchas cosas.

Pienso que lo que he logrado me permitirá alcanzar lo que espero hacer en el futuro.

$\mathrm{DE}$

Mis objetivos en la vida han sido más una fuente de satisfacción que de frustración para mí.

PV

Me gusta la mayor parte de los aspectos de mi personalidad.

0 Tengo clara la dirección y el objetivo de mi vida.

En general, con el tiempo siento que sigo aprendiendo más sobre mí mismo.

PV

$\mathrm{CP}$

22 No he experimentado muchas relaciones cercanas y de confianza.

RD

Sé que puedo confiar en mis amigos, y ellos saben que pueden confiar en mí.

$\mathrm{RD}$

Cuando pienso en ello, realmente con los años no he mejorado mucho como persona.

$\mathrm{CP}$

27 Tengo la sensación de que con el tiempo me he desarrollado mucho como persona.

$\mathrm{CP}$

28 Para mí, la vida ha sido un proceso continuo de estudio, cambio y crecimiento.

$\mathrm{CP}$

30 Mi vida está bien como está, no quiero intentar nuevas formas de hacer las cosas.

$\mathrm{CP}$ 